\title{
CEREBR-IO: Actividad lúdica para la enseñanza y el fortalecimiento de la Investigación de Operaciones
}

CEREBR-IO: Playful activity for teaching and strengthening the subject Investigación de Operaciones

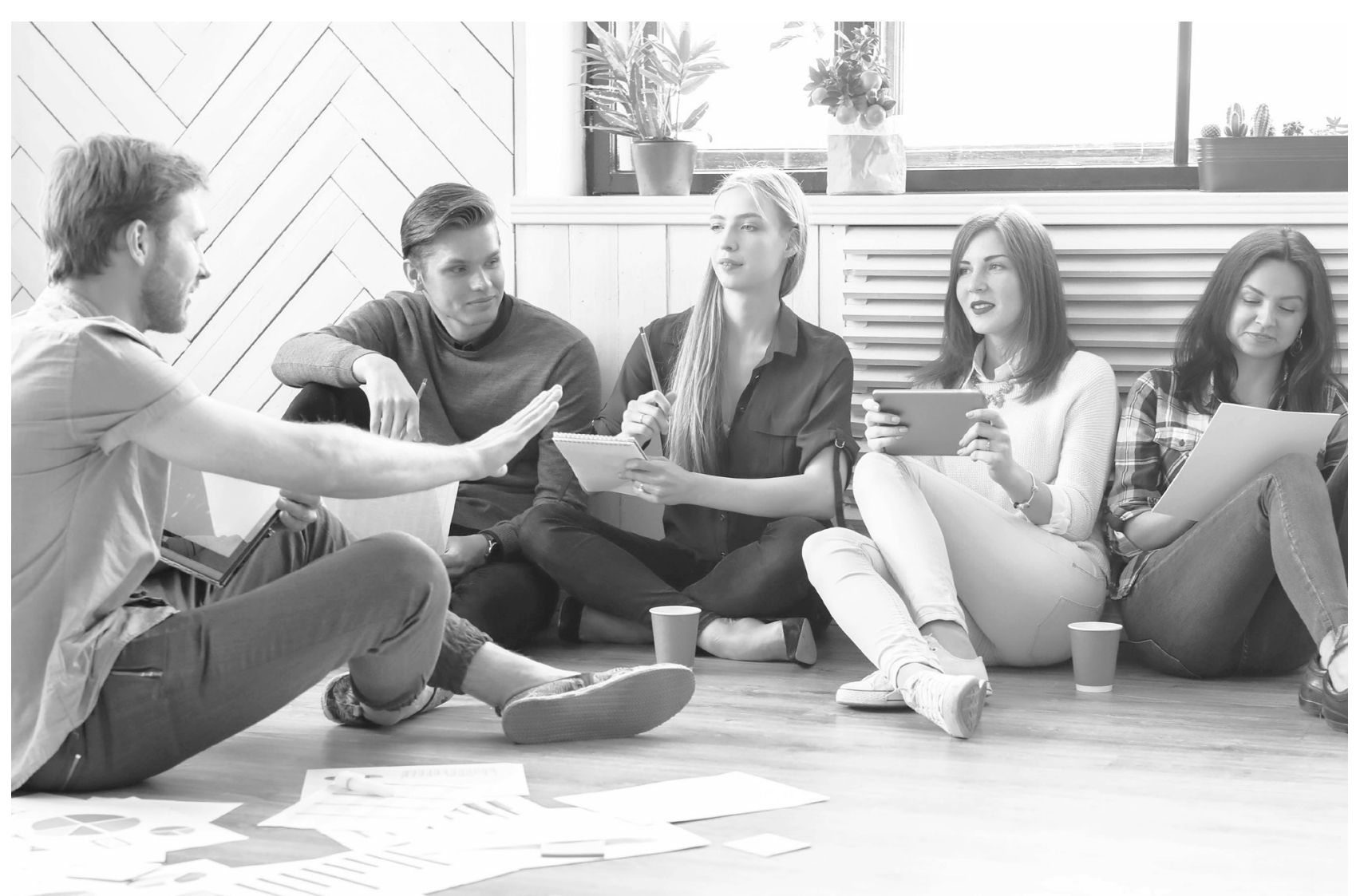




\title{
CEREBR-IO: Actividad lúdica para la enseñanza y el fortalecimiento de la Investigación de Operaciones ${ }^{1}$ \\ CEREBR-IO: Playful activity for teaching and strengthening the subject Investigación de Operaciones
}

\author{
Ariel Donaldo Caicedo Mora² ${ }^{2}$ Daniel Sebastián Benítez Agudelo ${ }^{3}$, Angie Marcela Ramírez Rubio ${ }^{4}$
}

Artículo recibido en abril 28 de 2020; artículo aceptado en junio 10 de 2020

Este artículo puede compartirse bajo la Licencia Creative Commons Atribución-NoComercial-Compartirlgual 4.0 Internacional y se referencia usando el siguiente formato: Caicedo, A. D., Benitez, D. S. y Ramirez, A. M. (2020). CEREBR-IO: Actividad lúdica para la enseñanza y el fortalecimiento de la Investigación de Operaciones. I+D Revista de Investigaciones, 15 (2), $148-162$.

DOI: https://doi.org/10.33304/revinv.v15n2-2020013

\begin{abstract}
Resumen
Este trabajo tiene como objetivo contribuir a la metodología de enseñanza-aprendizaje de la asignatura de Investigación de Operaciones I, a través del diseño y aplicación de una actividad lúdica. Para este fin, se utilizó el modelo de gamificación Canvas en el diseño. Para probar la pertinencia y la aceptación de la lúdica en el proceso de enseñanza- aprendizaje, tanto en los estudiantes como en el profesor, se hizo un estudio experimental, a través de la ejecución de una prueba piloto con una muestra de 16 estudiantes y la aplicación de un cuestionario al finalizar. Como resultado, se desarrolló CEREBR-IO, una actividad que permite apoyar el proceso de comprensión y apropiación de la programación lineal, especialmente, el proceso de modelación matemática y los conceptos teóricos clave. Esta actividad, de acuerdo con los comentarios de los estudiantes participantes en la validación, permite complementar de manera positiva las clases magistrales, haciéndolas más estimulantes y llamativas, en comparación con las clases tradicionales.
\end{abstract}

Palabras clave: Aprendizaje activo, Investigación de Operaciones, lúdica, programación lineal.

\begin{abstract}
This work aims to contribute to the teaching-learning methodology of the subject Investigación de Operaciones I,

${ }^{1}$ Artículo de investigación, de enfoque mixto, resultado de un trabajo de grado en curso, perteneciente al área ciencias sociales e ingeniería, subárea modelación y simulación y educación, desarrollado en el grupo de investigación GINNOVA, programa de Ingeniería Industrial, Universidad de Ibagué (Ibagué, Colombia). Dirección: carrera 22 calle 67 B, Av. Ambalá, Ibagué - 730002, Colombia. PBX: +57 (8) 2760010 . Fecha de inicio: febrero de 2020. Fecha de finalización: septiembre de 2020.

${ }^{2}$ Estudiante de Ingeniería Industrial, Universidad de Ibagué. Vinculado al programa de Ingeniería Industrial, Universidad de Ibagué (Ibagué, Colombia). Dirección: carrera 22 calle 67. PBX: +57(8) 2760010. ORCID ID: https://orcid.org/0000-0002-5006-7874. Correo electrónico institucional: 2320162055@estudiantesunibague.edu.co.

${ }^{3}$ Estudiante de Ingeniería Industrial, Universidad de Ibagué. Vinculado al programa de Ingeniería Industrial, Universidad de Ibagué (Ibagué, Colombia). Dirección: carrera 22 calle 67, PBX: +57(8) 2760010. ORCID ID: https://orcid.org/0000-0001-8661-9362. Correo electrónico institucional: 2320162021@estudiantesunibague.edu.co

${ }^{4}$ Especialista en Evaluación y Desarrollo de Proyectos, Universidad del Rosario. Vinculada al Grupo de investigación GINNOVA, programa de Ingeniería Industrial, Universidad de Ibagué (Ibagué, Colombia). Dirección: carrera 22 calle 67, PBX: +57(8) 2760010. ORCID ID: https://orcid. org/0000-0001-6390-4849. Correo electrónico institucional: angie.ramirez@unibague.edu.co
\end{abstract}


through the design and application of a playful activity. To this end, the Gamification Canvas model was used in the design. To test the relevance and acceptance of play in the teaching-learning process in both students and teachers, an experimental study was carried out through the execution of a pilot test with a sample of 16 students and the application of a questionnaire upon completion. As a result, "CEREBR-IO" was developed, an activity that supports the process of understanding and appropriating linear programming, especially the process of mathematical modeling and key theoretical concepts, which, according to the comments of the participating students in the validation, it allows to complement the masterclasses in a positive way, making them more stimulating and attractive compared to the traditional classes.

Keywords: Active learning, Investigación de Operaciones, playful, linear programming.

\section{Introducción}

En la actualidad, un $61 \%$ de la comunidad educativa considera que los alumnos no logran alcanzar las competencias necesarias para una vida laboral, debido principalmente a las limitaciones existentes en el aula de clases para aplicar de forma práctica el conocimiento teórico adquirido durante la carrera (Semana, 2015). Por esta razón, día a día se exploran nuevas alternativas de enseñanza en busca de mejorar la experiencia de aprendizaje. Una de las estrategias que ha venido creciendo en popularidad es el desarrollo de actividades lúdicas en materias que resultan poco prácticas o difíciles de comprender para los estudiantes. Estas actividades permiten complementar el desarrollo de las competencias profesionales deseadas, ya que refuerzan conocimientos y habilidades tales como la resolución de problemas, la colaboración y la comunicación (Moyano et al., 2019).

La lúdica ha sido considerada, desde diferentes ámbitos de la educación superior, como una metodología estratégica de enseñanza creativa, pues propicia escenarios favorables y estimulantes para promover el aprendizaje, ya que, en este tipo de actividades, los estudiantes tienen una participación activa dentro de su proceso de construcción del conocimiento, y pueden así asimilar las diferentes temáticas de los programas académicos (Torres, 2019).

En el campo de estudio de la Investigación de Operaciones, se hace necesaria la implementación de diversas estrategias pedagógicas que permitan fortalecer los procesos de abstracción matemática. Para los estudiantes, resulta complejo formular modelos matemáticos que permitan plasmar problemas del contexto real. En el caso de este estudio, se ha identificado que, desde el año 2017, aproximadamente un $21,35 \%$ de los estudiantes ha perdido la asignatura de Investigación de Operaciones I. Para solventar este problema, en otras universidades se han diseñado actividades lúdicas de aplicación de modelos y algoritmos lineales, tanto en formulación de modelos de programación lineal como en sus fundamentos teóricos.
Por ejemplo, la lúdica ¿Te atreves a brillar con la I.O.?, que consiste en el fortalecimiento de los conocimientos teóricos de la Investigación de Operaciones y de la capacidad de trabajo en equipo (Arévalo et al., 2019). Otra lúdica diseñada para trabajo en equipo es El caso del fabricante y el transportador, en donde el estudiante debe decidir cuáles y cuántos elementos son necesarios para construir diversos artículos y así obtener el máximo de ganancias; todo esto apoyado por el uso de la herramienta informática de Excel (Rivera y Pinzón, 2006). Así mismo, la lúdica El comercio de canicas: Herramienta de apoyo para la enseñanza-aprendizaje práctico de la programación lineal, en la que los estudiantes deben tomar decisiones para minimizar costos y maximizar utilidades bajo un escenario de mercado, en el que se espera introducir dos nuevos productos (canicas grandes y pequeñas), y donde cada uno tiene un costo diferente de transporte y publicidad, así como también una utilidad distinta en el mercado en el que se venda $(A \circ B)$, todo esto con el fin de complementar la clase magistral de programación lineal (PL) (Pérez et al., 2010).

A partir de la situación presentada, surgió la siguiente pregunta de investigación: ¿Cómo se pueden fortalecer los conceptos clave y reforzar el proceso de modelamiento matemático en la asignatura Investigación de Operaciones I en la carrera de Ingeniería Industrial de una universidad de la ciudad de Ibagué? Para dar respuesta a este interrogante, se planteó como objetivo general diseñar una estrategia de aprendizaje activo con enfoque lúdico que permita apoyar y complementar los conceptos teóricos y prácticos de la asignatura Investigación de Operaciones I.

Para cumplir con este objetivo, se diseñaron los siguientes objetivos específicos: proponer una actividad lúdica que permita reforzar la experiencia de aprendizaje en las aulas de clase con base en un juego interactivo; evaluar la actividad diseñada mediante una encuesta poslúdica realizada en una muestra seleccionada a conveniencia de estudiantes del curso de Investigación de Operaciones I, y establecer los beneficios de la actividad lúdica y sus puntos de mejora. 
Para dar cumplimiento al sistema de objetivos propuesto surgió la lúdica CEREBR-IO. Esta se diferencia de las lúdicas previamente identificadas esencialmente en la combinación de ejercicios prácticos y teóricos en un tablero innovador que incentiva el trabajo en equipo y la competencia entre los participantes. Esta actividad tiene como finalidad que los estudiantes reconozcan la importancia del papel de la formulación de modelos de programación lineal en la toma de decisiones para el cumplimiento de las metas organizacionales, de modo que recuerden y fortalezcan los conocimientos teóricos y prácticos de Investigación de Operaciones I, por medio de un tablero educativo y didáctico (ver Anexo I).

De acuerdo con la pregunta investigación planteada anteriormente, se realizó la siguiente hipótesis causal: la actividad de aprendizaje activo con enfoque lúdico CEREBRIO refuerza los conceptos clave y fortalece el proceso de formulación de modelos de programación lineal en la asignatura del núcleo profesional de Ingeniería Industrial: Investigación de Operaciones I.

\section{Marco referencial}

Teniendo en cuenta que este artículo se centra en generar una actividad con enfoque lúdico como refuerzo didáctico para las clases magistrales de Investigación de Operaciones I, será necesario plantear algunos parámetros que sirvan de ejes conceptuales para tener claridad de los componentes del aprendizaje activo con un enfoque lúdico. Para empezar, es primordial hablar sobre aprendizaje, que se puede explicar mediante el modelo de las 3P (pronóstico, proceso y producto). Este modelo se define de la siguiente manera:

En el pronóstico, se consideran las condiciones previas del estudiante al enfrentarse a un conocimiento nuevo y el contexto de la enseñanza que se enmarca en el definir qué se enseña. La etapa de proceso está definida por las actividades de aprendizaje, que deben tener intencionalidad de desarrollar conocimiento profundo y significativo. Y en la etapa tres, o etapa de producto, se busca evidenciar los resultados de aprendizaje, en donde se definen los métodos para evaluar el grado en que los estudiantes alcanzaron los objetivos de aprendizaje y las competencias. (Biggs, 2006, p.45)

Adicional al aprendizaje, otro concepto esencial es el de lúdica, esto considerando que los entornos lúdicos potencian el aprendizaje, pues se basan en el principio del "aprender haciendo" (Rodríguez Cepeda, 2018). De acuerdo con Hérnandez Cruz (2015), los seres humanos aprendemos el $20 \%$ de lo que escuchamos, el $50 \%$ de lo que vemos y el $80 \%$ de lo que hacemos.

Según Echeverri y Gómez (2009), "la lúdica se refiere a la necesidad del ser humano, de comunicarse, sentir, expresarse y producir emociones orientadas hacia el entretenimiento, la diversión, el esparcimiento, que pueden llevar mejor conocimiento al actuar en medio de una situación real". La lúdica fomenta además el desarrollo psicosocial mediante la adquisición de herramientas y saberes que interactúan con el gozo, la creatividad y el conocimiento (Echeverri y Gómez, 2009).

Es pertinente mencionar además la utilización de los micromundos como herramienta de apoyo al desarrollo de la lúdica, ya que ofrecen trasladar al estudiante a un mundo excitante en el que puede poner a prueba sus capacidades y desarrollar sus propios modelos mentales (Galvis, 2013), puesto que se genera conocimiento a través de la transformación de las experiencias vividas (Rodríguez Cepeda, 2018). Además, al introducir un micromundo en la creación de la lúdica, se genera un medio eficiente para acercar al estudiante a un tipo de aprendizaje más dinámico, lo que favorece el interés, la motivación y el aprendizaje por los contenidos de la asignatura.

Por consiguiente, un micromundo permite al estudiante plantear, probar y poner en práctica sus ideas, buscando conceptos nuevos para interactuar y enriquecer su experiencia educativa (Davila y Calpa, 2016). Las actividades que relacionan al micromundo deben formar parte de metodologías de aprendizaje constructivo fundamentadas en la actividad misma del sujeto sobre el objeto de estudio, que, en este caso, va enfocado al entendimiento y análisis de la Investigación de Operaciones I.

Para el diseño de actividades lúdicas, la Institución Tecnológica de Monterrey de México ha desarrollado una metodología llamada "gamificación Canvas", en la que se definen los principales elementos de un juego, con el propósito de que los profesores puedan implementarlo en el aula, tales como metas y objetivos, reglas, narrativa, libertad de elegir, libertad para equivocarse, recompensas, retroalimentación, competencia, restricción de tiempo y progreso. Así mismo, describe los seis tipos de jugadores que pueden encontrarse en actividades gamificadas: exploradores, socializadores, pensadores, filántropos, triunfadores y revolucionarios (Escamilla et al., 2016).

Diversos autores plantean que la gamificación o la lúdica está conformada por dinámicas, mecánicas y componentes. La dinámica es el concepto del juego, la mecánica es el desarrollo del juego y el componente es la implementación específica de las dinámicas y mecánicas que abarca los avatares, puntos, rankings, niveles, entre otros (Acosta-Medina, et al., 2020; Ortiz-Colón et al., 2018).

En cuanto al campo de estudio de interés de este 
trabajo, es necesario mencionar que la Investigación de Operaciones es de gran importancia en el entorno empresarial, pues es allí donde se desenvolverán los estudiantes como futuros profesionales, y donde una gran cantidad de ingenieros, gerentes o CEO de todo tipo de empresas se ven enfrentados a tomar decisiones considerando diversas alternativas. Entre ellas, el apoyo en los métodos cuantitativos, como la Investigación de Operaciones I, que proporciona modelos y técnicas con los cuales se procesan datos y se generan resultados numéricos en apoyo a la toma de decisiones, lo que brinda una aproximación a la solución de un problema práctico. Para realizar un estudio de Investigación de Operaciones, se sugiere seguir la siguiente secuencia de pasos (Hiller y Lieberman, 2017):

1. Definición del problema de interés y recolección de datos relevantes

2. Formulación de un modelo matemático que represente el problema

3. Desarrollo de un procedimiento basado en computadora para derivar una solución para el problema a partir del modelo

4. Prueba del modelo y mejoramiento de acuerdo con las necesidades

5. Preparación para la aplicación del modelo prescrito por la administración

6. Implementación

Así, los modelos de programación lineal son una herramienta cuantitativa para la toma de decisiones. Esta hace uso de la formulación de problemas de optimización de recursos y permite encontrar la solución óptima a situaciones reales que pueden ser expresadas con funciones y restricciones lineales (sistemas de inecuaciones lineales), optimizando una función objetivo también lineal (Chediak, 2013).

Los aspectos teóricos importantes en la asignatura de Investigación de Operaciones I se pueden resumir en la interpretación conceptual de los resultados obtenidos por medio de los modelos de programación lineal. Por ejemplo, la clasificación de las soluciones obtenidas como factibles y no factibles. La primera clasificación se refiere a la existencia de un conjunto de soluciones o valores que satisfacen las restricciones; de esta, a su vez, se desprende otra clasificación: solución única con solución múltiple (si existe más de una solución) y con solución no acotada (cuando no existe límite para la función objetivo). La segunda se presenta cuando existe un conjunto de soluciones que no cumple al menos una de las restricciones; esto también es conocido como problemas sin solución (Chediak, 2013).

\section{Metodología}

La lúdica de CEREBR-IO nace a partir de la necesidad de generar un nuevo proceso de aprendizaje para los estudiantes que cursan la materia Investigación de Operaciones I. Esta se basa principalmente en la formulación de modelos de programación lineal y sus conceptos teóricos. Como primera fase, se realizó la socialización de la idea de la lúdica con estudiantes del Semillero de Aplicación de Lúdicas en Ingeniería (SALI) de la Universidad de Ibagué y profesores del programa de Ingeniería Industrial, con el propósito de conocer la claridad del juego y hacerlo más emocionante y llamativo.

Como segunda fase, y con las ideas de la lúdica más claras, se llevó a cabo un proceso en el que los integrantes del semillero (estudiantes y profesores) tomaron el rol de jugador. Esta actividad consistió en imaginar las necesidades que pueden presentar los jugadores durante la lúdica, así como definir los objetivos de aprendizaje que se espera lograr a través del juego, y transformarlos en una idea creativa y constructiva que parte de los conocimientos básicos del estudiante al momento de jugarlo.

En la tercera fase, se desplegó la estructura del juego mediante el lienzo de gamificación Canvas (ver Anexo II), que permite plasmar las mejores ideas y los propósitos más importantes que se quieren lograr en la lúdica. Este lienzo de gamificación es una adaptación del Business Model Canvas propuesto por el Instituto Tecnológico y de Estudios Superiores de Monterrey.

En la fase final se desarrolló un ejercicio práctico de validación, con estudiantes de la asignatura Operación de Investigaciones I pertenecientes a una universidad de la ciudad de lbagué.

\section{Tipo de estudio}

El presente estudio es de tipo descriptivo, enfoque mixto y de carácter experimental, utilizando una metodología de tipo aplicada; es decir, que se encuentra centrada en encontrar mecanismos o estrategias que permitan lograr un objetivo concreto, que, para efectos de este estudio, consisten en generar una herramienta de tipo lúdico que permita servir de apoyo a las clases de la materia de Investigación de Operaciones I. Es de corte transversal, debido a que se llevará a cabo una serie de pruebas en un momento y lugar específico. En primer lugar, frente a un comité evaluativo conformado por dos profesores del programa de Ingeniería Industrial, con experiencia de tres años en el diseño de actividades lúdicas, y 10 estudiantes de ingeniería industrial de quinto, sexto y séptimo semestre pertenecientes al semillero SALI. Posteriormente, se realizó una prueba piloto con un grupo de 16 estudiantes, todo esto con el propósito de recolectar información que permita, además de mejorar la lúdica, evaluar el impacto generado por parte de esta en los alumnos. 


\section{Participantes}

La selección de los participantes del estudio se realizó por medio de un muestreo por conveniencia, debido al poco tiempo que toma realizarlo, su gran accesibilidad y su alto índice de participación, lo que facilita que los sujetos sean colaborativos (Mcmillan y Schumacher, 2005). Los sujetos debían cumplir con las siguientes características: ser estudiantes de la facultad de ingeniería y estar cursando la materia Investigación de Operaciones I.

De igual forma, para desarrollar el juego se consideran las siguientes características:

- Conocimiento básico en formulación de programación lineal

- Comprensión de la estructura de la formulación de modelos matemáticos

- Aprendizaje básico teórico de la Investigación de Operaciones

De esta forma, se obtuvo una muestra de 16 estudiantes participantes entre 18 y 25 años, quienes estaban cursando la asignatura de Investigación de Operaciones I, pertenecientes a los programas de Ingeniería Industrial e Ingeniería de Sistemas, y que participaron de forma voluntaria en el desarrollo de la prueba piloto de la lúdica CEREBR-IO.

\section{Instrumento}

En el proceso de validación de la lúdica, se aplicó un cuestionario como instrumento de recolección de información (ver Anexo III). Este fue desarrollado a partir del trabajo de Torres et al. (2019), con el propósito de recolectar evidencia física de la percepción de la lúdica por parte de los participantes en el proceso de aprendizaje-enseñanza, y evaluar así las fortalezas y puntos de mejora de la actividad diseñada.

\section{Resultados}

\section{Estructura de la lúdica}

A continuación, se explicará el paso a paso de las dimensiones que conforman la lúdica propuesta.

Objetivo: Para los jugadores, el objetivo es completar el recorrido, obtener la mayor cantidad de tesoros y superar a los demás equipos en el camino al conocimiento. Por otro lado, el objetivo pedagógico que persigue el juego es desarrollar las competencias de los estudiantes en la modelación matemática, de forma que comprendan la formulación de problemas de programación lineal con todos sus componentes.
Perfil de jugadores: Los jugadores pueden ser estudiantes de la facultad de ingeniería que se encuentren cursando la asignatura Investigación de Operaciones I, o personas que tengan conocimientos básicos en el área. Los jugadores que se espera tener en la lúdica son de tipo colaborativo, ya que juegan en beneficio de una interacción social trabajando de forma conjunta; pensadores, aquellos que se encargan de solucionar problemas, incógnitas o retos; por último, los de tipo triunfador, que cuentan con el deseo de ganar y superar todos los retos presentes en el juego.

Comportamientos esperados: El comportamiento esperado durante la lúdica principalmente se basa en que los alumnos tengan una buena actitud de participación, que se involucren en el juego y que identifiquen de manera más clara cada una de las dimensiones de la formulación y los conceptos básicos de la programación lineal. De igual forma, se espera que los estudiantes puedan adquirir o reforzar los conocimientos básicos necesarios en la asignatura de Investigación de Operaciones I.

Componentes: Para el desarrollo de la lúdica se conforman cuatro equipos de mínimo dos estudiantes. También, se requiere un tablero didáctico en forma de cerebro, similar a un parqués, en el cual se utilizan tesoros (monedas de oro), avatares, que representan a cada equipo, y una variedad de retos (preguntas abiertas y cerradas del caso y propias de la materia).

Mecánica: Para avanzar en el tablero, se necesita lanzar un dado didáctico por turnos (ver Anexo IV). En el tablero se deberá superar diversos obstáculos que permiten poner a prueba y fortalecer los conocimientos y el trabajo en equipo, a través de la solución de preguntas abiertas y cerradas, tanto de modelación matemática como de teoría propia de la materia Investigación de Operaciones I. Al superar los obstáculos, se desea obtener el mayor número de tesoros, ya que el equipo que logre completar todo el recorrido con la mayor cantidad de monedas será el que se titule vencedor de CEREBR-IO.

Dinámica: La dinámica del juego consiste en que los participantes que conforman los equipos se involucren dentro de un micromundo enfocado en la solución de problemas de la vida real. Esta narrativa, que se plantea dentro del tablero didáctico, permite sumergir a los participantes en el mundo de CEREBR-IO, motivándolos a enfrentar y superar los diversos retos que encontrarán dentro del juego.

En este sentido, el objetivo de la lúdica es que cada integrante de los diferentes equipos refuerce y ponga a prueba sus conocimientos adquiridos en programación lineal, así como también en aspectos clave de la 
Investigación de Operaciones I, siguiendo la temática del juego, de acuerdo como se explica a continuación.

Al momento de inicio de la lúdica, a todos los equipos (máximo cuatro para este ejercicio; cada uno integrado por dos personas) se les dará un mismo caso (problema de programación lineal) basado en la idea principal del ejercicio 1.14: "La dieta" (Chediak, 2013) (ver Anexo V). Los equipos deberán analizar y resolver este caso en cinco minutos. Cada equipo se identificará con un clan del planeta CEREBR-IO representado por un avatar (ficha) de color diferente.

El juego establece que los equipos avancen a través de un tablero didáctico, utilizando para ello un dado ilustrado, que indicará si pierde el turno, avanza o retrocede cierta cantidad de casillas. Es importante tener en cuenta que los elementos del dado son el cerebro creativo, que indica que el equipo puede avanzar la cantidad de casillas igual al número de cerebros creativos que haya obtenido (2, 3, 4 o 5); el cerebro con sueño, indica que el equipo avanza solo una casilla (1), y el cerebro cansado, significa que el equipo debe retroceder una casilla.

Los equipos obtendrán recompensas (monedas de oro), a medida que acierten las preguntas (tanto del caso como de preguntas aleatorias) dependiendo de la casilla en la que caigan. Dentro del tablero didáctico, se encontrarán diferentes retos de acuerdo con la figura correspondiente, como se muestra en la Tabla 1.

Tabla 1

\section{Retos del tablero didáctico}

Descripción
$\begin{aligned} & \text { Genera las preguntas correspondientes del } \\ & \text { caso. Si se responde bien, el equipo gana una } \\ & \text { moneda de oro; de no ser así, retrocede a la } \\ & \text { casilla anterior sin generar ningún efecto (ver } \\ & \text { Anexo VI). }\end{aligned}$

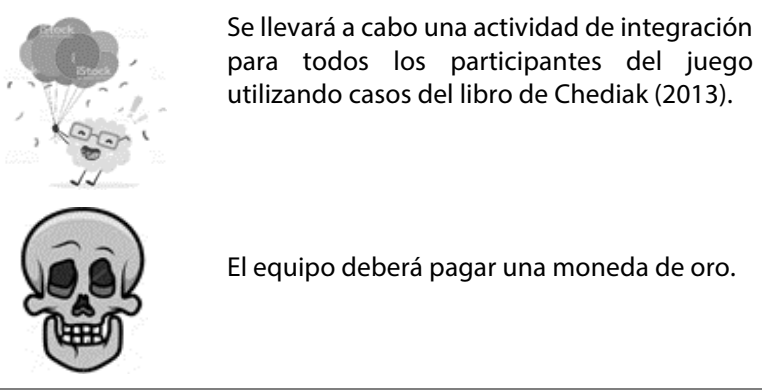

Fuente: Autores. Nota: las imágenes que se presentan en esta tabla se obtuvieron de Freepik.com $(2014,2019)$.

Cuando dos equipos se encuentren en la misma casilla, se generará un duelo entre equipos. En este duelo se llevará a cabo una serie de juegos tradicionales, como, por ejemplo, "piedra papel y tijera", para decidir qué equipo se queda en la casilla y cuál retrocede un puesto (ver Figura 1).

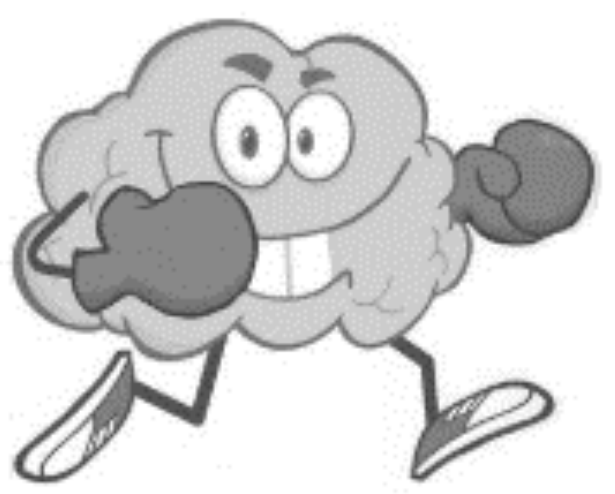

Figura 1. Duelo entre equipos. Fuente: Freepik.com (2020).

\section{Reglas}

- En caso de que el equipo tenga que retroceder casillas, solo lo hará máximo hasta la casilla de inicio. De ser así, el equipo que regrese al inicio deberá arrojar un dado de parqués, y todos los otros equipos podrán avanzar la cantidad de casillas igual a la que indique el dado.

- En caso de que el equipo tenga que retroceder o avanzar casillas y termine en una casilla con algún reto del tablero didáctico, este paso no deberá ser realizado en ese momento.

- En caso de que el equipo tenga que contestar preguntas abiertas, cerradas o del caso, dispondrá de 120 segundos para responder; si no las responde en el tiempo determinado, retrocederá una casilla.

- El equipo ganador será aquel que logre completar el recorrido del tablero y que obtenga la mayor cantidad de monedas de oro. 
- En caso de que otro equipo tenga más monedas que el equipo que completó el recorrido, este podrá retarlo a una pregunta a muerte. Si el equipo retado contesta de forma incorrecta, tendrá que ubicarse en la misma posición del equipo retador. En caso de que el equipo retado conteste bien, el equipo que lo retó tendrá que darle todas sus monedas y quedarse con solo una, y el equipo retado será el ganador del juego.

Gestión: Para realizar el seguimiento y monitoreo de la lúdica, se asignará un moderador por equipo. Este se encargará de apoyar y monitorear el juego, y también de observar las acciones de cada uno los participantes y comprobar si sus respuestas son correctas o incorrectas.

\section{Riesgos potenciales:}

- Existe el riesgo de que algunos de los integrantes de los equipos no tengan conocimiento que les permita solucionar el problema del reto

- Falta de comprensión de las reglas

- Tiempo insuficiente para culminar el juego

- Monotonía

Para contrarrestar estos riesgos, los moderadores deben mantener la motivación en los participantes, y el equipo que dirige el juego debe estar dispuesto a clarificar las dudas que vayan surgiendo durante la actividad. Así mismo, controlará el tiempo, de forma que haya una buena utilización de este recurso.

Estética: Los elementos que darán identidad visual y estética al juego se pueden observar en las Figuras 2 y 3 .

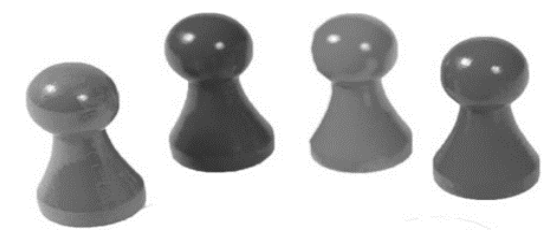

Figura 2. Avatares. Fuente: Freepik.com (2016).

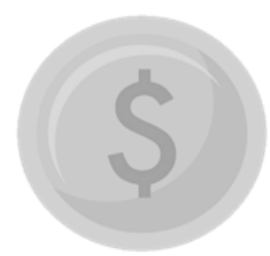

Figura 3. Tesoros. Fuente: Freepik.com (2018).

Finalizada la lúdica, se genera un espacio de realimentación donde el moderador da respuesta a las inquietudes de los participantes sobre los principales problemas que tuvieron al momento de plantear los modelos de programación lineal. De igual forma, este espacio permitirá que los estudiantes sean más conscientes de sus fortalezas y debilidades en la formulación de modelos de programación lineal.

\section{Materiales de la lúdica}

Para el desarrollo de la actividad, se requiere un área libre de $6 \mathrm{~m} \times 8 \mathrm{~m}$, dotada de un computador, un video beam y una mesa de aproximadamente $2,5 \mathrm{~m} \times 1 \mathrm{~m}$. Igualmente, se requieren los siguientes materiales:

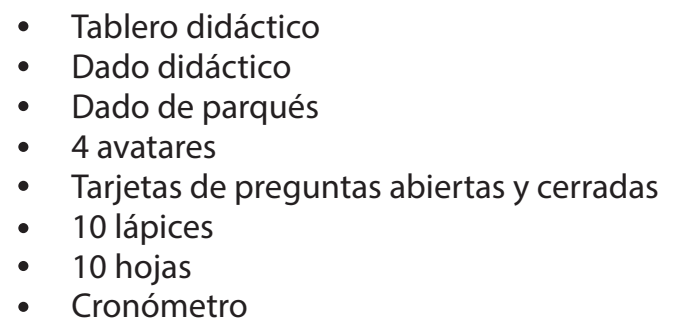

\section{Socialización comité evaluativo (profesores y estudiantes pertenecientes a SALI)}

El día 21 de febrero de 2020 se llevó a cabo la socialización de la lúdica CEREBR-IO frente al semillero de investigación SALI, dando a conocer la idea del juego, con todos sus componentes. Esta socialización permitió generar cambios que mejoraron el diseño de la lúdica, gracias a las correcciones y opiniones brindadas por los miembros de este comité, que se basaron principalmente en clarificar algunas reglas planteadas por los autores, la creación de preguntas a realizar durante la lúdica y la corrección o mejoramiento de algunas preguntas ya existentes.

\section{Desarrollo de prueba piloto}

El día 9 de marzo de 2020 se ejecutó la prueba piloto de la lúdica, con 16 estudiantes de Investigación de Operaciones I, como se evidencia en el Anexo VIII. Posterior al desarrollo de la lúdica, se aplicó un cuestionario con 10 preguntas de carácter cuantitativo en escala Likert (en donde 1 significa en total desacuerdo; y 5 en total acuerdo) y tres preguntas abiertas de carácter cualitativo, según lo indican Torres et al. (2019). Esto permitió estimar la aceptación y el impacto generado por parte de la lúdica en los estudiantes. En los Anexos IX y $\mathrm{X}$ se pueden observar los resultados obtenidos, respectivamente.

De acuerdo con la información recolectada, se encuentra que el $96,34 \%$ de los participantes está totalmente de acuerdo en que la actividad pone a prueba los 
conocimientos adquiridos en clase. Por otra parte, la pregunta que presentó menor porcentaje fue la de "Las instrucciones y reglas del juego son claras y fáciles de comprender", con el $73,43 \%$ de los participantes que estaba de acuerdo, lo que puede deberse a que algunos estudiantes expresaron que eran demasiadas reglas para memorizarlas de momento. Para esto, se podría implementar un manual o libro de reglas con el que cada equipo cuente en el transcurso del juego; aun así, cabe resaltar que esta fue la única por debajo del $80 \%$.

De igual forma, es pertinente resaltar que de las preguntas 1 y 8 hechas en el cuestionario relacionadas con la generación, el refuerzo y el entendimiento de la formulación de modelos matemáticos, un promedio de $69,45 \%$ de los participantes estuvo totalmente de acuerdo con la pertinencia de la lúdica en el refuerzo de las clases magistrales. Finalmente, en promedio se obtuvo un $89,24 \%$ de favorabilidad frente a la aceptación de la lúdica por parte de la muestra.

Así mismo, se realizó el análisis cualitativo con las preguntas abiertas, que corresponden a ¿Qué aspecto recomendaría para mejorar la lúdica?, ¿Qué le gustó de la lúdica? y ¿Qué no le gustó de la lúdica? Para esto, se agruparon las respuestas por su número de repetición, teniendo en cuenta las de mayor frecuencia, como se observa en el cuestionario.

Del análisis se pudo determinar que 5 de los 16 estudiantes recomiendan ampliar el tiempo establecido para dar respuesta a las preguntas. Como solución a esta sugerencia, se puede realizar una reestructuración de los límites de tiempo utilizados en la lúdica. Así mismo, se halló que uno de cada cinco estudiantes considera que la lúdica fortalece la competitividad. Por otra parte, un cuarto de la muestra expresó que hace falta más explicación en los casos y preguntas, para lo cual se esperaría mejorar la participación de los moderadores, con el fin de que los estudiantes sientan el proceso de acompañamiento durante la actividad, y puedan fortalecer sus debilidades en la asignatura.

\section{Conclusiones}

Este artículo sugiere que el uso de actividades lúdicas ayuda a fortalecer los conocimientos básicos de los estudiantes en la materia Investigación de Operaciones I, ya que los alumnos sienten que las clases apoyadas con métodos educativos como la lúdica resultan ser más estimulantes, agradables y llaman su atención de mayor manera que la clase magistral. Este hallazgo está en concordancia con lo que plantea Torres (2019), quien indica que este tipo de estrategias didácticas contribuye a la motivación de estudiantes y docentes dentro de la ejecución de actividades en el aula; idea apoyada por diversos autores (Acosta-Medina et al., 2020). Además, esta lúdica genera y apoya la participación del trabajo en equipo, al igual que sucede en otras lúdicas, como la de Pérez et al. (2010) o la de Arévalo et al. (2019).

Del análisis de los datos recolectados en la aplicación del instrumento, tanto cuantitativos como cualitativos, se concluye que la lúdica presentada permitió reforzar los conocimientos en la formulación de modelos matemáticos de programación lineal, así como también los conceptos teóricos básicos de la Investigación de Operaciones I, confirmando la pertinencia y el beneficio de la lúdica en la materia para el desarrollo del aprendizaje de los estudiantes, y está en línea con lo expuesto por Torres (2019) en su documento.

De igual forma, teniendo en cuenta los resultados del análisis cualitativo, es fundamental un ajuste en el tiempo que tienen los participantes para dar respuesta a los retos que el juego conlleva, así como ajustar o reorganizar la manera en la que se explican los casos y preguntas de la lúdica.

Por otra parte, se puede mencionar que se logró el objetivo de generar una herramienta de apoyo a las clases magistrales de la materia de Investigación de Operaciones. Los profesores pueden utilizar esta herramienta como complemento en el proceso de aprendizaje-enseñanza de sus cátedras, a fin de cambiar el ambiente teórico por uno más práctico.

Cabe mencionar que el diseño de la lúdica es muy genérico, debido a las bases en las que se fundamentó, como lo es el diseño de un juego de parqués, lo que permite que este sea aplicado en otras materias con tan solo realizar unos pequeños cambios, como cambiar las preguntas y casos necesarios para el juego. Esto sirve no solo de apoyo al método de enseñanza-aprendizaje de investigaciones de operaciones, sino de cualquier otra asignatura.

\section{Agradecimientos}

Especial agradecimiento a los estudiantes del programa de Ingeniería Industrial de la Universidad de Ibagué que participaron en el desarrollo de esta actividad, CEREBR-IO. Igualmente, al semillero SALI (Semillero de Aplicación de Lúdicas en Ingeniería) de la Universidad de lbagué, por el apoyo a un mejor entendimiento de la lúdica.

\section{Referencias}

Acosta-Medina, J. K., Torres-Barreto, M. L. y AlvarezMelgarejo, M. (2020). Literature Mapping About Gamification in the Teaching And Learning Processes. Revista ESPACIOS, 41(11), 26. https://www. revistaespacios.com/a20v41n11/20411126.html 
Acosta-Medina, J. K., Torres-Barreto, M. L., AlvarezMelgarejo, M. y Paba-Medina, M. C. (2020). Gamificación en el ámbito educativo: Un análisis bibliométrico. I+ D Revista de Investigaciones, 15(1), 28-36. https://doi.org/10.33304/revinv.v15n12020003

Arévalo, A., Arias, C., Calpa, J., Pérez, N. y Sánchez, C. (2019). ¿Te atreves a brillar con la I.O.? Séptimo Encuentro de La Red Iddeal. https://peewah.co/ events/septimo-encuentro-nacional-red-iddeal

Biggs, J. (2006). Calidad del aprendizaje universitario. (2.a ed) Narcea. https://barajasvictor.files.wordpress. com/2014/05/libro-j-biggs.pdf

Chediak, F. A. (2013). Investigación de Operaciones. Volumen I. (3.a ed.). Universidad de Ibagué.

Davila, J. y Calpa, J. (2016). Desarrollo de actividades de aprendizaje activo con enfoque lúdico para el complemento del proceso de enseñanza aprendizaje de la línea académica administración de operaciones (Universidad Autónoma de Occidente). https://sitios.uao. edu.co/docentes/wp-content/uploads/sites/26/2016/07/ InformeFinaldeproyectodeinvestigacion.docx-2.pdf

Echeverri, J. y Gómez, J. (2009). Lo lúdico como componente de lo pedagógico, la cultura, el juego y la dimensión humana. En Marco teórico investigación sobre la dimensión Lúdica del maestro en formación 2009. http://blog.utp.edu.co/areaderecreacionpcdyr/ files/2012/07/LO-LUDICO-COMO-COMPONENTE-DELO-PEDAGOGICO.pdf

Escamilla, J., Fuerte, K., Venegas, E., Fernández, K., Elizondo, J. y Román, R. (2016). Gamificación. Observatorio de Innovación Educativa Del Tecnológico de Monterrey, 1-36. http://eduteka.icesi.edu.co/pdfdir/edutrendsgamificacion.pdf

Freepik.com. (2014). Dibujo de calavera vector gratuito. https://www.freepik.es/vector-gratis/dibujocalavera_724076.htm

Freepik.com. (2016). Peones del ajedrez de colores colocados en fila Foto gratis. https://www.freepik. es/foto-gratis/peones-ajedrez-colores-colocadosfila_959172.htm

Freepik.com. (2018). Dólar vector gratuito. https://www. freepik.es/vector-gratis/dolar_2900482.htm

Freepik.com. (2019). Conjunto de dibujos animados de cerebro vector premium. https://www.freepik. es/vector-premium/conjunto-dibujos-animadoscerebro_5096282.htm

Freepik.com. (2020). Cerebro corriendo con guantes. https://www.freepik.es/search?dates=any\&forma $\mathrm{t}=$ search\&page $=1$ \&query=cerebro corriendo con guantes\&sort=popular

Galvis, A. (2013). Ambientes educativos CLIC -creativos, lúdicos, interactivos y colaborativos-para aprender en la era de la información. https://books.google.com. $\mathrm{co} /$ books?hl=es\&lr=\&id=ry2RDwAAQBAJ\&oi=fnd \& $p g=P A 63 \& d q=u s o+d e+m i c r o m u n d o s+e n+l a+l u d i c$ a\&ots=-5IvAzkhfl\&sig=ueIROVnnzoZSkgxSSBpmEel
FQ6I\&redir_esc $=y \# v=$ onepage \&q\&f=false

Hérnandez Cruz, H. (2015). La lúdica en el aula de ingeniería. Revisión de experiencias. Revista Ingeniería, Matemáticas y Ciencias de la Información, 2(3), 67-71. https://urepublicana.edu.co/ojs/index. php/ingenieria/article/view/239

Hiller, F. S. y Lieberman, G. J. (2017). Introducción a la investigación de operaciones (Novena). https://doi. org/10.2307/j.ctt2111g6q.5

Mcmillan, J. y Schumacher, S. (2005). Diseños y métodos de investigación cuantitativa. In Investigación educativa. (5.a ed). Pearson. https://des-for.infd.edu.ar/sitio/ upload/McMillan_J._H._Schumacher_S._2005. Investigacion_educativa_5_ed..pdf

Moyano, B. C., Morató, M. M. y Santos, J. (2019). La gamificación en la educación superior. Aspectos a considerar para una buena aplicación. Pedagogías Emergentes en la Sociedad Digital, 21.

Ortiz-Colón, A.-M., Jordán, J. y Agredal, M. (2018). Gamificación en educación: una panorámica sobre el estado de la cuestión. Educação e Pesquisa, 44(0). https://doi.org/10.1590/s1678-4634201844173773

Pérez, J., Jaramillo, P. y Moreno, L. (2010). El comercio de canicas: herramienta de apoyo para la enseñanzaaprendizaje práctico de la programación lineal. Revista Facultad de Ingeniería UCV, 25(1), 4352. http://ve.scielo.org/scielo.php?script=sci_ arttext\&pid=S0798-40652010000100005

Rivera, R. D. y Pinzón, T. M. (2006). La lúdica en la Investigación de Operaciones: El caso del fabricante y el transportador. Páginas: Revista Académica $e$ Institucional de La UCPR, (74).

Rodríguez Cepeda, R. (2018). Los modelos de aprendizaje de Kolb, Honey y Mumford: implicaciones para la educación en ciencias. Sophia, 14(1), 51-64. https:// doi.org/10.18634/sophiaj.14v.1i.698

Semana. (2015). Los universitarios no están preparados para el mercado laboral. https://www.semana.com/ educacion/articulo/posibilidades-laborales-de-losuniversitarios/447900-3

Torres, L. M. (2019). La importancia de la lúdica como estrategia didáctica en el proceso de enseñanza y aprendizaje en la educación superior [trabajo de especialización, Universidad Militar Nueva Granada]. Repositorio institucional, https://repository. unimilitar.edu.co/bitstream/handle/10654/31929/ TorresForeroLuisaMaria 2019.pdf?sequence $=1$

Torres, M., Paz, K. y Salazar, F. G. (2019). Métodos de recolección de datos para una investigación. Boletín Electrónico, 3, 1-21. https://www.academia. edu/16383192/URL_03_BAS01 


\section{Anexo I: Tablero de juego}

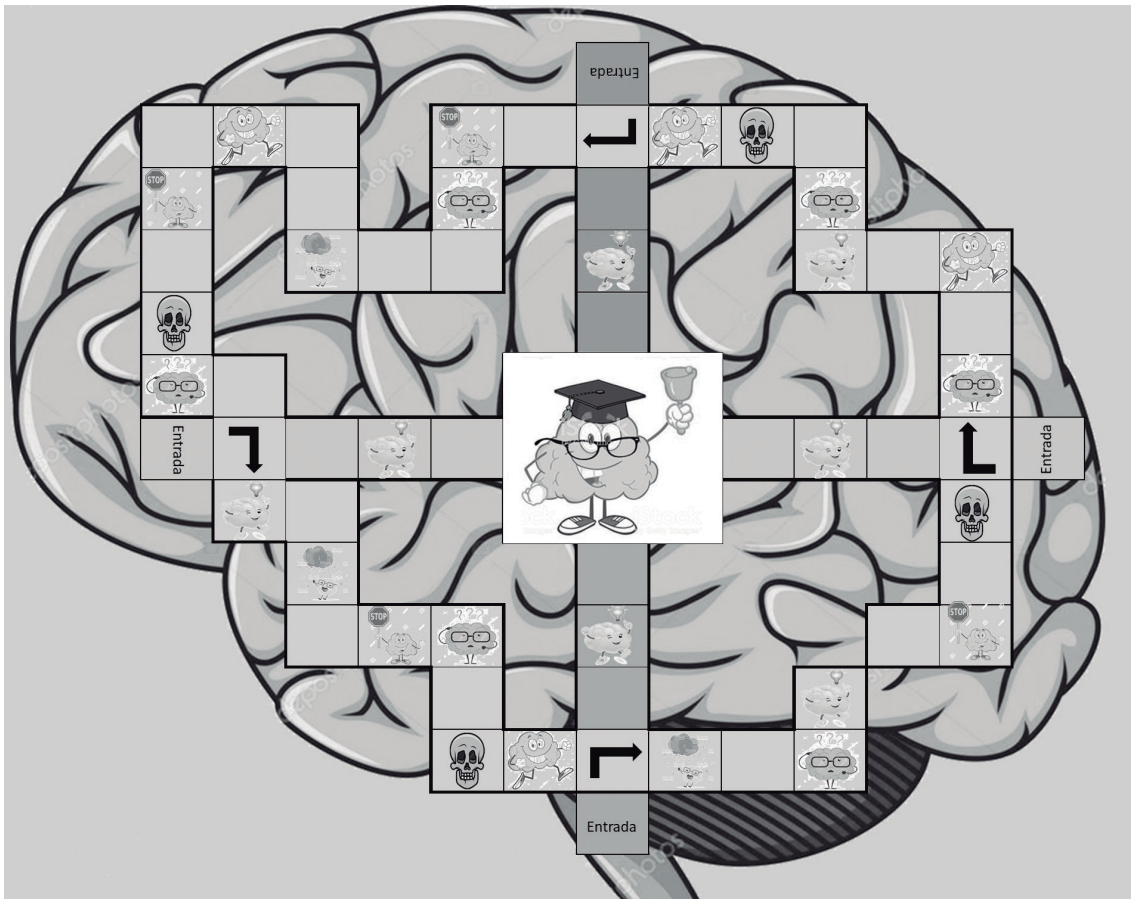

Figura 4. Tablero de juego. Fuente: Autores.

\section{Anexo II: Modelo de gamificación Canvas}

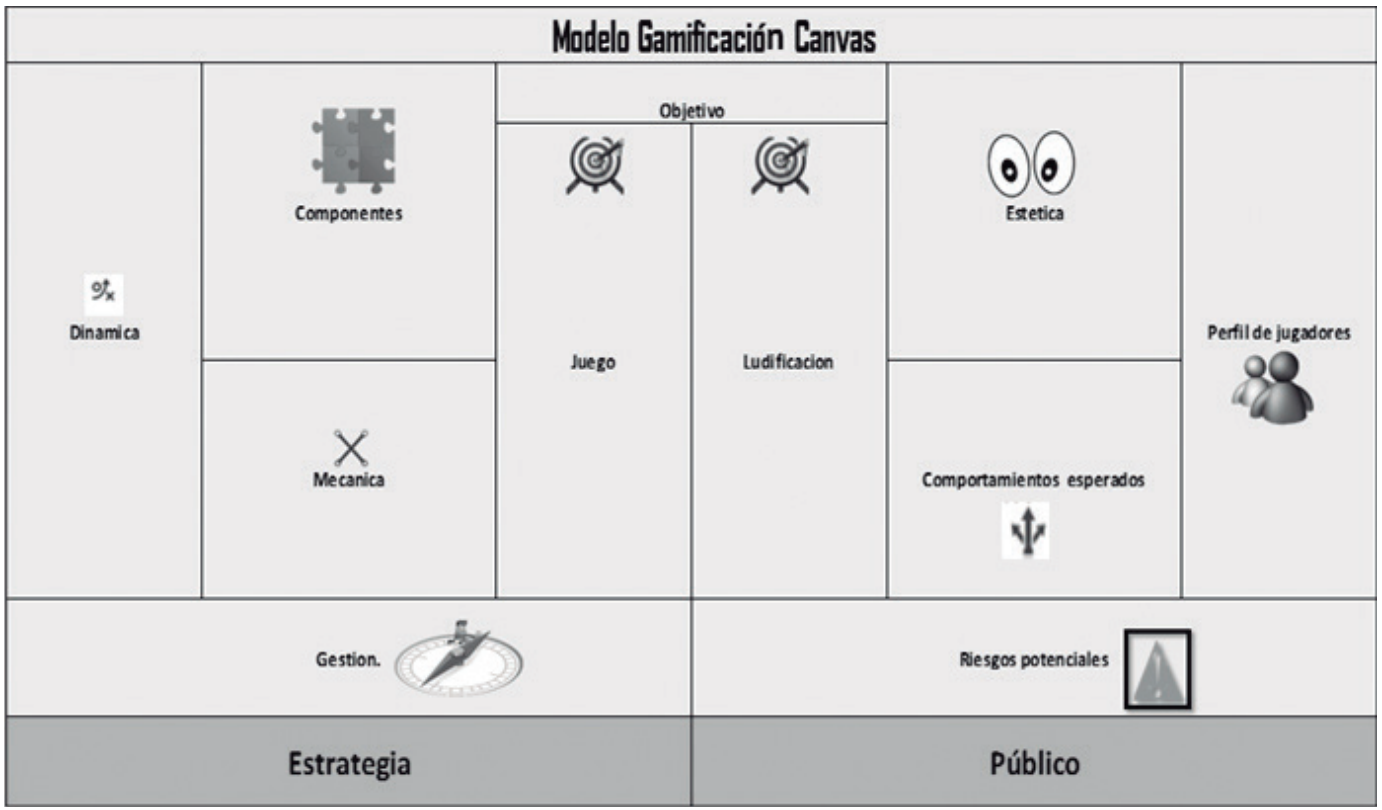

Figura 5. Lienzo de gamificación Canvas. Fuente: Tecnológico de Monterrey.

\section{Anexo III: Cuestionario poslúdica}

\section{Realimentación de la actividad}

Apreciados estudiantes, la siguiente encuesta tiene como objetivo conocer su opinión frente a la lúdica realizada. La información recolectada se utilizará solamente para fines académicos. 
A continuación, van a encontrar una lista de afirmaciones. Por favor calificar del 1 al 5, donde 1 es en total desacuerdo, y 5 en total de acuerdo. De igual forma encontrará una serie de preguntas abiertas, las cuales les solicitamos responder de la manera más honesta posible, de antemano agradecemos de toda su honestidad y colaboración.

Tabla 2

Preguntas del cuestionario de retroalimentación

\section{Afirmaciones}

El juego reforzó el tema de formulación de modelos de programación lineal y los conceptos base de la asignatura de Investigación de Operaciones I.

\begin{tabular}{l}
\hline La actividad pone a prueba los conocimientos adquiridos en clase. \\
\hline El juego fomenta el trabajo en equipo. \\
\hline Las instrucciones y reglas del juego son claras y fáciles de comprender. \\
\hline La experiencia en el juego fue satisfactoria. \\
\hline El juego reforzó el entendimiento de la formulación de modelos matemáticos. \\
\hline Los materiales utilizados dentro del juego fueron los apropiados. \\
\hline Este tipo de actividades aportan a la generación y refuerzo del conocimiento. \\
\hline El juego puso a prueba la interpretación de modelos matemáticos. \\
\hline Los juegos deben estar inmersos en la enseñanza de materias como Investigación de Operaciones.
\end{tabular}

1. ¿Qué aspectos recomendaría para mejorar la lúdica?

2. ¿Qué le gusto de la lúdica?

3. ¿Qué no le gustó de la lúdica?

Fuente: Autores.

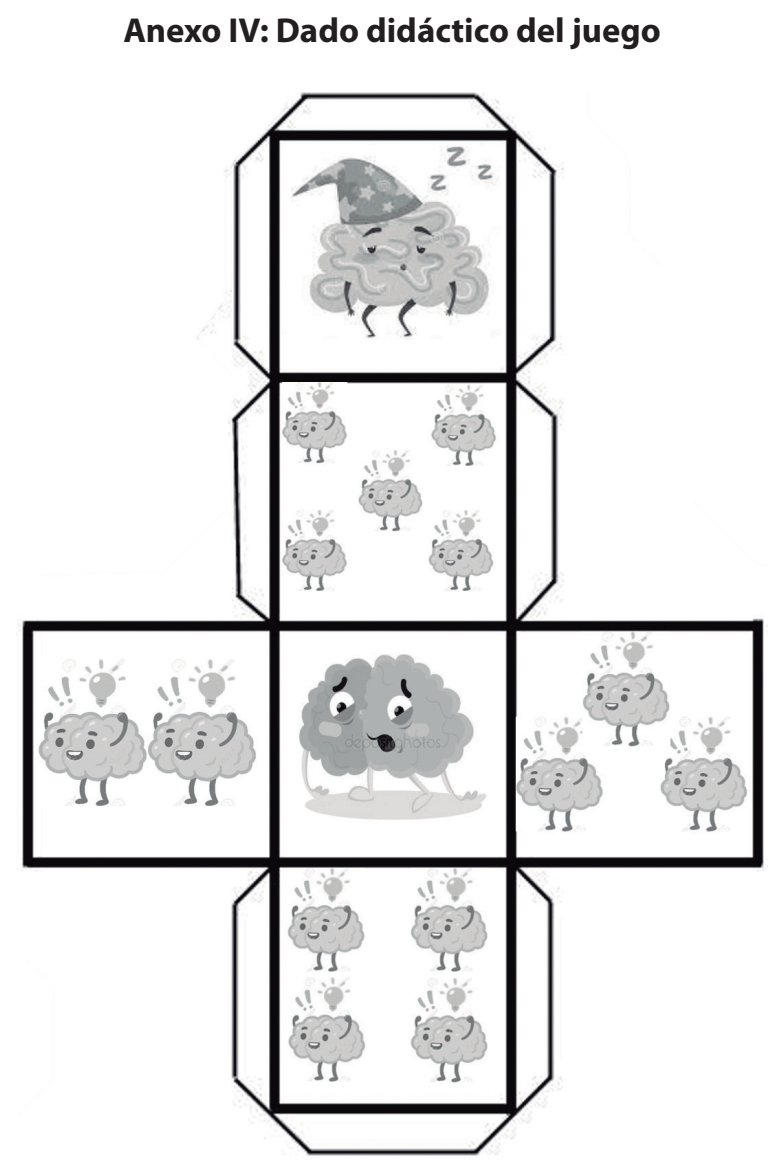

Figura 6. Dado didáctico. Fuente: Autores. 


\section{Anexo V: Caso principal}

Un instituto de bienestar familiar está realizando un estudio para determinar los alimentos que deberían ser incluidos en las meriendas que se les brinda a los niños en los hogares infantiles.

Tabla 3

Datos caso principal de la lúdica

\begin{tabular}{|c|c|c|c|c|c|c|c|}
\hline Alimento & Calorías & Proteína $(\mathrm{g})$ & Carbohidratos $(\mathrm{g})$ & Grasa $(\mathrm{g})$ & Calorías de grasa $(\mathrm{g})$ & Azúcar $(\mathrm{g})$ & \$/unidad \\
\hline Pan (1 tajada) & 62 & 2 & 12 & 1 & 9 & 1 & 100 \\
\hline Jamón (1 tajada) & 25 & 3 & 2 & 1 & 9 & 0 & 500 \\
\hline Queso (1 tajada) & 80 & 7 & 0 & 6 & 42 & 0 & 400 \\
\hline Yogurt (1 taza) & 80 & 7 & 12 & 0 & 0 & 11 & 800 \\
\hline Galletas (1 unidad) & 30 & 0 & 4 & 1 & 9 & 3 & 200 \\
\hline Jugo (1 taza) & 88 & 0 & 22 & 0 & 0 & 21 & 700 \\
\hline
\end{tabular}

Fuente: Autores.

Los requerimientos nutricionales son los siguientes: la merienda debe tener como mínimo un aporte nutricional de 400 calorías y como máximo 600 . No más del $25 \%$ de las calorías debe venir de la grasa. Cada niño debe consumir al menos 12 gramos de proteína y como máximo 40 gramos de carbohidrato. Es claro que si se le suministra un sándwich como merienda, se necesitan dos tajadas de pan, y por cuestiones de sabor, también se requiere que el número de tajadas de queso sea al menos el doble de las tajadas de jamón. Es necesario que se le brinde al menos una taza de jugo. Se desea seleccionar las opciones de alimentos a brindar de la merienda, de forma que se minimice el costo asociado y que se cumplan los requerimientos.

\section{Anexo VI: Preguntas propias del caso}

Tabla 4

Preguntas cerradas

¿La variable de decisión que está generando su caso es la siguiente?

¿Qué tipo de parámetros se deberían tener en cuenta para el desarrollo de su caso?

¿Cuál es la restricción que determina el mínimo número de calorías? Siendo X1= Tajadas de pan, X2= Tajadas de jamón, X3=Tajadas de queso, $\mathrm{X} 4=$ Tazas de yogurt, $\mathrm{X} 5=$ Galletas, $\mathrm{X} 6=$ Tazas de jugo.

¿Cuál es la restricción que determina el máximo número de gramos de proteína? Siendo X1= Tajadas de pan, X2= Tajadas de jamón, X3=Tajadas de queso, $\mathrm{X} 4=$ =Tazas de yogurt, $\mathrm{X} 5=$ Galletas, $\mathrm{X} 6=$ Tazas de jugo.

¿Según el caso de la restricción determina el número de calorías de grasa en la merienda? Siendo X1= Tajadas de pan, X2=Tajadas de jamón, X3=Tajadas de queso, X4=Tazas de yogurt, X5= Galletas, X6= Tazas de jugo.

¿Según el caso de la restricción determina la relación entre número de tajadas de jamón y queso? Siendo X1= Tajadas de pan, X2= Tajadas de jamón, X3=Tajadas de queso, X4=Tazas de yogurt, X5= Galletas, X6= Tazas de jugo.

Fuente: Autores.

\section{Anexo VII: Preguntas teóricas}

Tabla 5

Preguntas abiertas

1. ¿Para qué se utiliza la condición de no negatividad en un problema de Investigación de Operaciones?
2. ¿Cuántas soluciones óptimas puede tener un problema? 


\begin{tabular}{|c|c|}
\hline 3. ¿Qué es una solución factible? & $\begin{array}{c}\text { 4. ¿Cuándo se dice que un problema tiene múltiples soluciones } \\
\text { óptimas? }\end{array}$ \\
\hline 5. ¿Cuándo se considera que la solución no es factible? & $\begin{array}{c}\text { 6. ¿Qué tipos de solución puede haber en un modelo de } \\
\text { programación lineal? }\end{array}$ \\
\hline 7. ¿Qué significa que una solución es óptima? & 8. ¿Usualmente cuántas soluciones factibles tiene un problema? \\
\hline $\begin{array}{l}\text { 9. ¿A qué hacen referencias las restricciones de su caso? } \\
\text { (Mencione al menos 3) }\end{array}$ & $\begin{array}{l}\text { 10. ¿Para qué se utiliza el término Xij al definir una variable de } \\
\text { decisión? }\end{array}$ \\
\hline $\begin{array}{c}\text { 11. ¿Qué le ofrece la Investigación de Operaciones al ingeniero } \\
\text { industrial en su vida laboral? }\end{array}$ & $\begin{array}{l}\text { 12. En un problema general, de acuerdo con sus } \\
\text { conocimientos, ¿cómo interpretaría usted la siguiente } \\
\text { solución óptima? } X 1: 2 \times 2: 6 z=\$ 36.000\end{array}$ \\
\hline 13. ¿Cuáles son las partes de un modelo de programación lineal? & 14. ¿Qué significa que una restricción sea de tipo =? \\
\hline 15. ¿Para qué sirve el software Gusek? & $\begin{array}{c}\text { 16. ¿Considera que todas las restricciones deben estar expresadas } \\
\text { en función de las variables de decisión?? }\end{array}$ \\
\hline $\begin{array}{l}\text { 17. Cuando se habla de una restricción de capacidad u oferta, ¿a } \\
\text { qué se hace referencia? }\end{array}$ & $\begin{array}{l}\text { 18. ¿Qué se debe tener en cuenta a la hora de crear un conjunto en } \\
\text { Gusek? }\end{array}$ \\
\hline $\begin{array}{c}\text { 19. ¿Cómo expresaría su función objetivo con notación } \\
\text { matemática? }\end{array}$ & 20. Con base en su caso, ¿qué parámetros crearía? \\
\hline 21. Con base en su caso, ¿qué conjuntos crearía? & 22. ¿Cómo expresaría sus restricciones con notación matemática? \\
\hline
\end{tabular}

Fuente: Autores.

\section{Anexo VIII: Evidencia fotográfica}

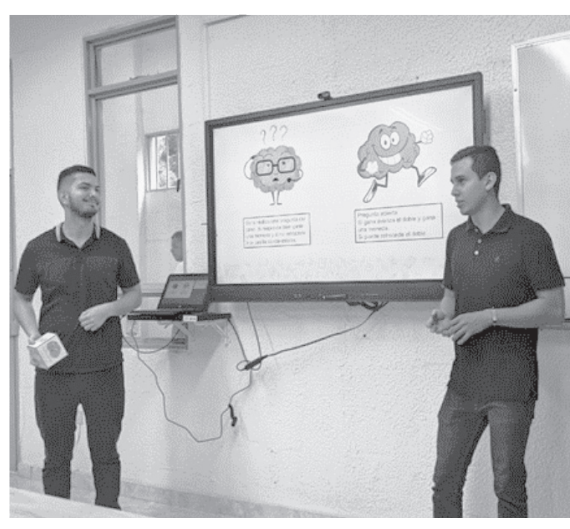

Figura 7. Presentación de la lúdica.

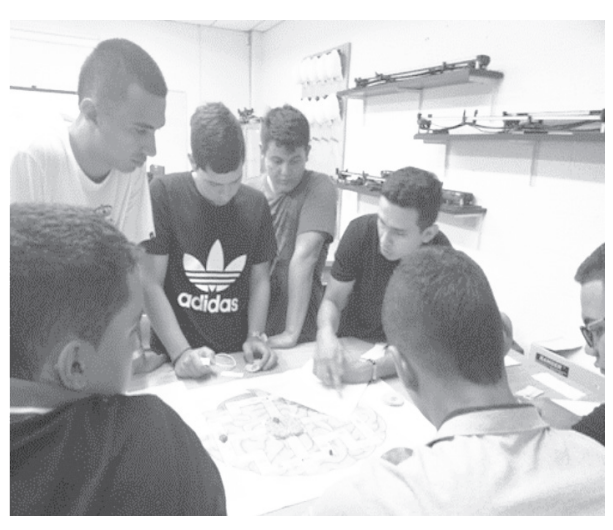

Figura 8. Realimentación de la lúdica.

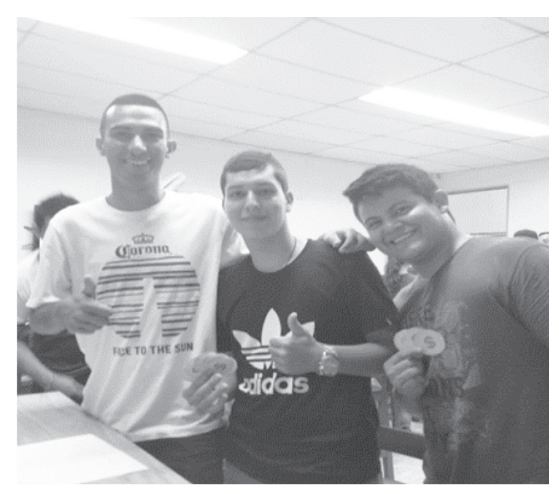

Figura 9. Ganadores de la lúdica. 


\section{Anexo IX: Resultados de preguntas en escala de Likert}

Tabla 6

Resultados de medición de percepción y aceptación cuantitativa de la lúdica

\begin{tabular}{|c|c|c|c|c|c|c|c|c|c|c|}
\hline & $\begin{array}{c}\text { Afirmación } \\
1\end{array}$ & $\begin{array}{c}\text { Afirmación } \\
2\end{array}$ & $\begin{array}{c}\text { Afirmación } \\
3\end{array}$ & $\begin{array}{c}\text { Afirmación } \\
4\end{array}$ & $\begin{array}{c}\text { Afirmación } \\
5\end{array}$ & $\begin{array}{c}\text { Afirmación } \\
6\end{array}$ & $\begin{array}{c}\text { Afirmación } \\
7\end{array}$ & $\begin{array}{c}\text { Afirmación } \\
8\end{array}$ & $\begin{array}{c}\text { Afirmación } \\
9\end{array}$ & $\begin{array}{c}\text { Afirmación } \\
10\end{array}$ \\
\hline Est.1 & 4 & 5 & 4 & 4 & 4 & 4 & 3 & 4 & 5 & 4 \\
\hline Est.1 & 5 & 5 & 5 & 5 & 5 & 5 & 5 & 5 & 5 & 5 \\
\hline Est.1 & 5 & 5 & 5 & 1 & 1 & 5 & 3 & 5 & 5 & 5 \\
\hline Est.1 & 3 & 4 & 4 & 3 & 4 & 2 & 3 & 2 & 3 & 5 \\
\hline Est.1 & 5 & 5 & 5 & 4 & 4 & 4 & 3 & 2 & 3 & 5 \\
\hline Est.1 & 4 & 5 & 5 & 3 & 5 & 5 & 5 & 5 & 4 & 4 \\
\hline Est.1 & 5 & 5 & 5 & 3 & 4 & 3 & 4 & 4 & 3 & 4 \\
\hline Est.1 & 4 & 4 & 3 & 4 & 4 & 5 & 5 & 4 & 3 & 4 \\
\hline Est.1 & 5 & 5 & 5 & 4 & 5 & 5 & 5 & 5 & 5 & 5 \\
\hline Est.1 & 5 & 5 & 5 & 4 & 5 & 5 & 5 & 5 & 5 & 5 \\
\hline Est.1 & 5 & 5 & 5 & 4 & 5 & 5 & 5 & 5 & 5 & 5 \\
\hline Est.1 & 5 & 5 & 5 & 3 & 4 & 5 & 5 & 5 & 5 & 5 \\
\hline Est.1 & 5 & 4 & 4 & 3 & 4 & 5 & 3 & 4 & 4 & 4 \\
\hline Est.1 & 5 & 5 & 4 & 5 & 5 & 4 & 5 & 5 & 5 & 5 \\
\hline Est.1 & 5 & 5 & 5 & 4 & 5 & 5 & 5 & 5 & 5 & 5 \\
\hline Est.1 & 5 & 5 & 4 & 4 & 5 & 5 & 5 & 5 & 5 & 5 \\
\hline Promedio & 4,69 & 4,81 & 4,56 & 3,63 & 4,31 & 4,5 & 4,31 & 4,38 & 4,38 & 4,69 \\
\hline$\%$ afir & $94 \%$ & $96 \%$ & $91 \%$ & $73 \%$ & $86 \%$ & $90 \%$ & $86 \%$ & $88 \%$ & $88 \%$ & $94 \%$ \\
\hline
\end{tabular}

Fuente: Autores.

\section{Anexo X: Resultados de preguntas abiertas}

Tabla 7

Resultados de medición, de percepción y aceptación cualitativa de la lúdica

\begin{tabular}{|c|c|c|}
\hline Preguntas abiertas & Respuestas con mayor repetición & Estudiantes \\
\hline ¿Qué aspectos recomendaría para mejorar la lúdica? & Dar más tiempo para las respuestas & 5 \\
\hline ¿Qué le gusto de la lúdica? & La competitividad & 3 \\
\hline ¿Qué no le gusto de la lúdica? & Falta más explicación en los casos y preguntas & 4 \\
\hline
\end{tabular}

Fuente: Autores. 